\title{
Puerarin enhances proliferation and osteoblastic differentiation of human bone marrow stromal cells via a nitric oxide/cyclic guanosine monophosphate signaling pathway
}

\author{
HAIHONG LV ${ }^{1}$, TUANJIE CHE ${ }^{2}$, XULEI TANG ${ }^{1}$, LIJUAN LIU ${ }^{1}$ and JIANGUO CHENG ${ }^{1}$ \\ ${ }^{1}$ Department of Endocrinology and Metabolism, The First Hospital of Lanzhou University; \\ ${ }^{2}$ Baiyuan Gene Technology Co. Ltd, Lanzhou, Gansu 730000, P.R. China
}

Received June 30, 2014; Accepted March 12, 2015

DOI: $10.3892 / \mathrm{mmr} .2015 .3647$

\begin{abstract}
Puerarin, a major active isoflavone extracted from the Traditional Chinese Medicine Radix Puerariae, has been studied for its comprehensive biological effects. However, to date, its effect on bone formation and the underlying mechanism of action have not been well investigated. The present study investigated the effect of puerarin on cell proliferation and osteoblastic maturation in cultured human bone marrow stromal cells (hBMSC) in vitro. Puerarin (2.5-100 $\mu \mathrm{M})$ increased hBMSC growth in a dose-dependent manner, as indicated by an MTT assay, and stimulated osteoblastic maturation as indicated by assessment of alkaline phosphatase (ALP) activity, as well as calcium deposition into the extracellular matrix detected by alizarin red S staining. Furthermore, polymerase chain reaction analysis showed that the expression of osteoblastic markers, including Runt-related transcription factor 2/core-binding factor alpha 1, osterix and osteocalcin, were increased in hBMSCs following incubation with puerarin. Further experiments indicated that puerarin increased the nitric oxide (NO) production and cyclic guanosine monophosphate (cGMP) content in hBMSCs. The effects of puerarin were mimicked by $17 \beta$-estrodiol $\left(10^{-8} \mathrm{M}\right)$ and were abolished in the presence of estrogen receptor antagonist ICI182780 $\left(10^{-7} \mathrm{M}\right)$. A NO synthase inhibitor, Nx-nitro-L-arginine methylester $\left(6 \times 10^{-3} \mathrm{M}\right)$, significantly attenuated puerarin-induced increases in NO production and cGMP content, in parallel with
\end{abstract}

Correspondence to: Dr Haihong Lv, Department of Endocrinology and Metabolism, The First Hospital of Lanzhou University, 1 Donggang West Road, Lanzhou, Gansu 730000, P.R. China

E-mail: haihonglv@126.com

Abbreviations: NO, nitric oxide; cGMP, cyclic guanosine monophosphate; hBMSC, human bone marrow stromal cells; HRT, hormone replacement therapy; ALP, alkaline phosphatase; L-NAME, NX-nitro-L-arginine methyl ester

Key words: puerarin, human bone marrow stromal cells, bone formation, nitric oxide/cyclic guanosine monophosphate pathway a reduction of cell proliferation and osteoblastic differentiation as well as the expression of osteoblastic markers. These results suggested that puerarin may prevent osteoporosis by exerting stimulatory effects on bone formation and the NO/cGMP pathway, which has an important role in puerarin-induced hBMSC proliferation and osteoblastic differentiation.

\section{Introduction}

Osteoporosis associated with estrogen deficiency is among the most common diseases in post-menopausal women (1). After menopause, women undergo a sharp decline in bone mass, losing $\sim 10-15 \%$ of bone over a period of 5-10 years (2). Hormone replacement therapy (HRT) is able to effectively prevent post-menopausal osteoporosis and reduce the incidence of fractures (3). However, HRT also increases the risk of breast and endometrial cancer, in addition to other undesirable side effects (4). Since phytoestrogens extracted from traditional Chinese medicines are different from hormones used in hormone replacement therapy, they are potentially important in the prevention of postmenopausal osteoporosis.

Phytoestrogens are divided into three classes: Isoflavones, coumestans and lignans. Puerarin [7-hydroxy-3-(4-hydro xyphenyl)-1-benzopyran-4-one-8-( $\beta$-D-glucopyranoside]

(Fig. 1), the main isoflavone glycoside found in the root of Pueraria lobata (Willd.) Ohwi, has been used for various medicinal purposes in Traditional Chinese Medicine for thousands of years (5). Phytoestrogens, including isoflavones, are molecules of plant origin, and are structurally associated with the mammalian estrogen $17 \beta$-estradiol $(6,7)$. Modern pharmacological research has demonstrated that puerarin has key effects on prevention and treatment of cardiovascular diseases, osteoporosis, diabetes and obesity, menopausal symptoms, renal diseases and various cancers $(8,9)$. Interestingly, puerarin has estrogenic activity $(10,11)$. Studies have shown that Radix Puerariae prevents bone loss by growth hormone release in ovariectomized rats $(9,12)$. Puerarin has also been reported to have a stimulatory effect on bone formation and activation of the PI3K/Akt pathway, regulating cell proliferation in rat calvaria osteoblasts (13). However, the detailed mechanisms underlying the anabolic effects of puerarin on osteoblasts have remained elusive. 
Further studies have indicated that estrogen 2 (E2) stimulation of osteoblast proliferation and differentiation is mediated via the nitric oxide (NO)/NO synthase (NOS) pathway (14-16). NO release in osteoblastic cells increases cGMP formation by binding guanylate cyclase (GC), and the resulting cGMP signal regulates osteoblastic proliferation and differentiation $(17,18)$. It was also revealed that the GC/cGMP system has a key role in spasmolytic signaling mechanisms. Previous studies showed that the phytoestrogen genistein stimulates osteoblastic differentiation via NO/cGMP in bone marrow-derived mesenchymal stem cells (BMSCs) of mice in primary culture (19). Thus, puerarin may also act on bone cells through the NO/cGMP pathway.

In the present study, the in vitro effect of puerarin on proliferation and osteoblastic maturation of hBMSCs was investigated. Furthermore, the effects of puerarin on osteoblastic bone formation and NO/cGMP production were assessed. To elucidate the underlying mechanism, estrogen activity and the NO pathway were blocked by ICI182780 or Nx-nitro-L-arginine methyl ester (L-NAME), respectively. The present study suggested that puerarin stimulates osteoblastic bone formation, likely through activation of the NO/cGMP signaling pathway.

\section{Materials and methods}

Reagents. Puerarin (99\% purity), pronase E, ascorbic acid, $\beta$-glycerophosphate, $p$-nitrophenol, diethanolamine, $p$-nitrophenol phosphate (p-NPP), L-NAME, 3 -isobutyl-L-methylxanthine (IBMX) and dextran-charcoal were all purchased from Sigma-Aldrich (St. Louis, MO, USA). Alpha minimum essential medium ( $\alpha$-MEM), fetal bovine serum (FBS), penicillin-streptomycin solution, SDS and TRIzol reagent were obtained from GIBCO-BRL (Invitrogen Life Technologies, Grand Island, NY, USA). ICI182780 was purchased from Tocris Cookson Ltd. (Avonmouth, Bristol, UK). Tissue culture plastic dishes and flasks were purchased from Corning-Costar Co. (Corning, NY, USA). A nitrate/nitrite colorimetric assay kit was purchased from Sino-American Biotechnology Company (Beijing, China). Molecular biology reagents and enzymes, including Tris- $\mathrm{HCl}$ buffer, $0.1 \%$ (w/v) alizarin red $\mathrm{S}$ and alkaline phosphatase were purchased from Boehringer Ingelheim (Ingelheim, Germany); Bio-Rad Protein Assay kit; $0.1 \mathrm{~N} \mathrm{NaOH/0.1 \%} \mathrm{SDS;} 2.5 \%$ glutaraldehyde and $70 \%$ ethanol were purchased from Bio-Rad Laboratories, Inc., Hercules, CA, USA; A cyclic GMP immunoassay kit was purchased from R\&D systems, Inc. (Minneapolis, MN, USA). All other chemicals were of analytical grade and purchased from Shanghai Biotech Co., Ltd. (Shanghai, China).

Cell culture. Primary hBMSCs were obtained from ribs discarded at the time of open thoracotomy in patients without metabolic bone disease using a protocol approved by the First Hospital of Lanzhou University Ethics Committee. The patients consisted of 14 males and 6 females whose mean age was 38.2 \pm 2.3 years (range, 18-57 years), and written informed consent was obtained from each patient. Primary hBMSCs were isolated and cultured in DMEM/F12 medium (GIBCO-BRL) containing $10 \% \mathrm{FBS}, 100 \mathrm{U} / \mathrm{ml}$ penicillin and $100 \mu \mathrm{g} / \mathrm{ml}$ streptomycin. Cells were maintained at $37^{\circ} \mathrm{C}$ in a humidified $5 \%$

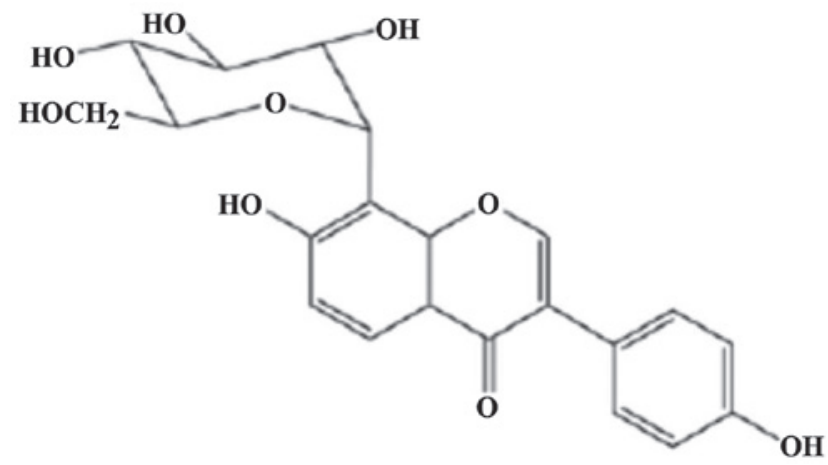

Figure 1. Chemical structure of puerarin.

$\mathrm{CO}_{2}$ incubator and identified as osteoblast-lineage by positive staining with alizarin red and alkaline phosphatase. Confluent (85-95\%) hBMSCs were washed twice with PBS prior to the experiments. Cells were then treated with a series of concentrations of puerarin $\left(2.5-100 \mu \mathrm{M}\right.$, dissolved in PBS) or $10^{-8} \mathrm{mM}$ $17 \beta$-estradiol (Sigma-Aldrich) in fresh medium containing $10 \%$ FBS for the indicated times.

MTT assay. Cell proliferation was determined by a colorimetric assay based on the ability of viable cells to metabolize MTT (Jiancheng Bioengineering Institute, Nanjing, China). MTT is a yellow tetrazolium salt which is reduced by the mitochondria of metabolically active cells to form a blue formazan dye precipitate that can be extracted using an organic solvent. Cells were seeded at a density of $2 \times 10^{3}$ cells/well in 96-well plates in DMEM/F12 medium with $20 \%$ FBS. After $48 \mathrm{~h}$, confluent (85-95\%) cells were cultured with various concentrations of puerarin or $17 \beta$-estradiol in serum-free DMEM/F12 medium for $48 \mathrm{~h}$, respectively. Three hours prior to the end of the cell incubation period, $10 \mu \mathrm{l} 0.5 \%$ MTT solution was added to each culture well. Following an additional 3-h incubation at $37^{\circ} \mathrm{C}$, the medium was removed and the formazan crystals were dissolved in $0.2 \mathrm{ml}$ dimethyl sulfoxide for $30 \mathrm{~min}$ at $37^{\circ} \mathrm{C}$. The optical density (OD) of each well was measured at $570 \mathrm{~nm}$ using a microplate reader (Spectra Max M2e Microplate Reader; Molecular Devices Corporation, Sunnyvale, CA, USA). Values are expressed as the percentage of the OD of the control cells.

Measurement of NO production. hBMSCs were grown in the differentiation medium [1.0\% Triton X-100, $2 \mathrm{mM} \mathrm{MgCl}$, DEA $10 \mathrm{ml}$ (Sino-American Biotechnology Company) and $8 \mathrm{mM}$ PNPP (Sigma-Aldrich)] and treated as indicated for 12 days. The culture media were collected, and nitrite production in the conditioned media $(\mathrm{CM})$ was measured using a nitrite chromometry assay kit in a modified Griess assay. Briefly, $100 \mu \mathrm{l} \mathrm{CM}$ or nitrite standards $(0-100 \mu \mathrm{M})$ were mixed with $100 \mu \mathrm{l}$ of Griess reagent (Sino-American Biotechnology Company). Absorbance was then measured at $530 \mathrm{~nm}$ against a blank prepared with distilled water, and the release of NO into the culture medium was evaluated as the nitrite concentration, which was determined from a standard curve.

Alkaline phosphatase (ALP) assay. ALP is known to be associated with bone metabolism and differentiation of osteo- 
blasts. ALP activity is one of the most common indicators of osteoblast differentiation and osteogenic properties. Following treatment with puerarin or $17 \beta$-estradiol $\left(10^{-8} \mathrm{M}\right)$ for $48 \mathrm{~h}$, hBMSCs were washed twice with PBS and lysed in $10 \mathrm{mM}$ Tris- $\mathrm{HCl}$ buffer (pH 7.6) containing $2 \mathrm{mM} \mathrm{MgCl}_{2}$ and $0.1 \%$ Triton X-100 on ice for $30 \mathrm{~min}$, then centrifuged at $18,000 \mathrm{x} \mathrm{g}$ for $10 \mathrm{~min}\left(\right.$ at $\left.4^{\circ} \mathrm{C}\right)$. The clear supernatant was stored frozen at $-20^{\circ} \mathrm{C}$ until use. Intracellular ALP was determined using an ALP kit (Jiancheng Bioengineering Institute, Nanjing, China). Briefly, $20 \mathrm{ml}$ of the diluted cell lysates were incubated in 96-well plates with $180 \mathrm{~m} 0.1 \mathrm{mM} \mathrm{NaHCO}-\mathrm{Na}_{2} \mathrm{CO}_{3}$ buffer (pH 10.0) containing $1.0 \%$ Triton $\mathrm{X}-100,2 \mathrm{mM} \mathrm{MgCl}_{2}$ and $8 \mathrm{mM} \mathrm{p}$-NPP for $30 \mathrm{~min}$ at $37^{\circ} \mathrm{C}$. The absorbance of p-nitrophenol liberated in the reactive solution was read at $450 \mathrm{~nm}$. $25 \mathrm{ml}$ of the diluted cell lysates was measured at $550 \mathrm{~nm}$ for total protein content using a bicinchoninic acid protein assay kit (Shanghai Biotech Co., Ltd.).

Mineral nodule formation assay. To examine the effect of puerarin on nodule formation, hBMSCs were incubated in 24-well plates for $24 \mathrm{~h}$ and treated with puerarin or $17 \beta$-estradiol $\left(10^{-8} \mathrm{M}\right)$ for 14 days. Cultures were fed every two days by replacing them with fresh medium (containing $10 \%$ FBS) and reagents. Cells were rinsed with PBS, fixed in $2.5 \%$ glutaraldehyde for $10 \mathrm{~min}$ and washed three times in $70 \%$ ethanol. Following drying for $20 \mathrm{~min}$, cells were stained with $01 \%$ (w/v) alizarin red S for $30 \mathrm{~min}$. Mineral nodules with a short diameter $>100 \mu \mathrm{m}$ in 10 fields were counted using a light microscope (Olympus BX5001; Olympus Optical Co. Ltd., Tokyo, Japan). Calcium deposition in mineralized nodules was assessed by a modification of the Wada procedure (20). The cultures were decalcified with $0.6 \mathrm{~N} \mathrm{HCl}$ for $24 \mathrm{~h}$. The calcium content in the $\mathrm{HCl}$ supernatant was determined by the $o$-cresolphthalein complexone method (21). After decalcification, the cultures were washed with PBS and solubilized with $0.1 \mathrm{~N} \mathrm{NaOH} / 0.1 \%$ SDS. Total protein content was measured with a Bio-Rad protein assay kit. The calcium content of the cell layer was normalized to the protein content.

Measurement of the accumulation of cGMP. Cells were grown in differentiation medium and treated with puerarin or $17 \beta$-estradiol $\left(10^{-8} \mathrm{M}\right)$ in the presence or absence of L-NAME $\left(6 \times 10^{-3} \mathrm{M}\right)$ or ICI182780 $\left(10^{-7} \mathrm{M}\right)$ for eight days. Following being washed with phenol-free $\alpha$-MEM, the cells were incubated with phenol-free $\alpha$-MEM supplemented with $5 \times 10^{-4} \mathrm{M}$ IBMX, a diesterase inhibitor, at $37^{\circ} \mathrm{C}$ for $15 \mathrm{~min}$. The reagents used for treatment were then added to the media as above and the cells were incubated for another hour. After incubation, the amount of cGMP in each sample was measured using a cGMP ELISA kit from R\&D Systems.

Reverse transcription quantitative polymerase chain reaction $(R T-q P C R)$. Total RNA was extracted from each six-culture well at the end of the incubation period (nodule formation) using RNA simple Total RNA kit [Tiangen Biotech (Beijing) Co., Beijing, China]. Reverse transcription of total RNA to cDNA was performed with SuperScript III First Strand Synthesis System for RT-PCR (Invitrogen Life Technologies, Carlsbad, CA, USA) in a MyCycler Thermal Cycler (Bio-Rad Laboratories, Inc.) following the manufacturer's instructions.
qPCR was performed using a LightCycler 480 SYBR Green I Master Mix (Roche Diagnostics GmbH, Mannheim, Germany) in a LightCycler 480 system (Roche Diagnostics). The PCR reaction was performed in a $20-\mu 1$ volume in a LightCycler 480 96-well plate and the cycling protocol was as follows: $95^{\circ} \mathrm{C}$ for $5 \mathrm{~min}$, followed by $45 \mathrm{PCR}$ cycles of $95^{\circ} \mathrm{C}$ for $5 \mathrm{sec}, 60^{\circ} \mathrm{C}$ for $15 \mathrm{sec}$ and $72^{\circ} \mathrm{C}$ for $20 \mathrm{sec}$. Dissociation curves were run after amplification to identify the specific PCR products. LightCycler 480 software, version 1.5 was employed to perform the relative quantification for the expression of target genes.

Specific primers for Runt-related transcription factor 2 (Runx2), osterix and osteocalcin mRNA were as follows: Runx2 mRNA forward, 5'-TGCTTCATTCGCCTCACAAA-3' and reverse, 5'-TTGCAGTCTTCCTGGAGAAAGTT-3'; osterix mRNA forward, 5'-CCTCTGCGGGACTCAACAAC-3' and reverse, 5'-TAAAGGGGGCTGGATAAGCAT-3'; osteocalcin mRNA forward, 5'-GGACTGTGACGAGTTGGCTG-3' and reverse, 5'-CCGTAGAAGCGCCGATAGG-3'. $\beta$-actin was used as the reference: $\beta$-actin mRNA forward, 5'-CGGTCA GGTCATCACTATCG-3' and reverse, 5'-TTCCATACCCAG GAAGGAAG-3'.

Statistical analysis. Statistical analysis was performed using GraphPad Prism version 5 software (GraphPad Software, Inc., La Jolla, CA, USA). All values were expressed as the mean \pm standard deviation and statistically analyzed by one-way analysis of variance. $\mathrm{P}<0.05$ was considered to indicate a statistically significant difference.

\section{Results}

Puerarin $(10 \mu M)$ stimulates $h B M S C$ proliferation. To evaluate the effect of puerarin on cell viability, an MTT assay was performed. hBMSCs were treated with puerarin for $48 \mathrm{~h}$ at various concentrations and the results are presented in Fig. 2. Puerarin at lower concentrations significantly increased cell growth of hBMSCs, with increases of $54.6 \pm 1.6 \%$ at $10 \mu \mathrm{M}$ and $39.1 \pm 1.3 \%$ at $25 \mu \mathrm{M}$, which were similar to those of $17 \beta$-estradiol (45.1 $\pm 1.1 \%$ ) (Fig. 2A). However, the concentration of $100 \mu \mathrm{M}$ had an obvious inhibition on osteoblastic growth, and cell viability was reduced to $79.1 \pm 2.3 \%(\mathrm{P}<0.05)$ of that of the control group, suggesting that its higher concentration may be cytotoxic in hBMSCs, as confirmed by light microscopy following trypan blue staining (20\% cell death; results not shown). As $10 \mu \mathrm{M}$ was the puerarin concentration which was most effective on osteoblastic differentiation and proliferation of hBMSC cultures, it was selected to be used in the subsequent experiments. The puerarin-induced increase in proliferation was completely abrogated in the presence of the estrogen inhibitor ICI182780 $\left(10^{-7} \mathrm{M}\right)$ or the NOS inhibitor L-NAME $\left(6 \times 10^{-3} \mathrm{M}\right)$. ICI182780 or L-NAME alone did not have any detectable effects (Fig. 2B).

Puerarin $(10 \mu \mathrm{M})$ enhances osteoblastic differentiation of hBMSCs. To determine whether puerarin altered ALP activity, a marker for the differentiation of hBMSCs, cellular ALP activity was measured following incubation with different concentrations of puerarin (Fig. 3). Puerarin had marked effects on ALP activity, which was increased by $34.65 \pm 1.4 \%$ and 

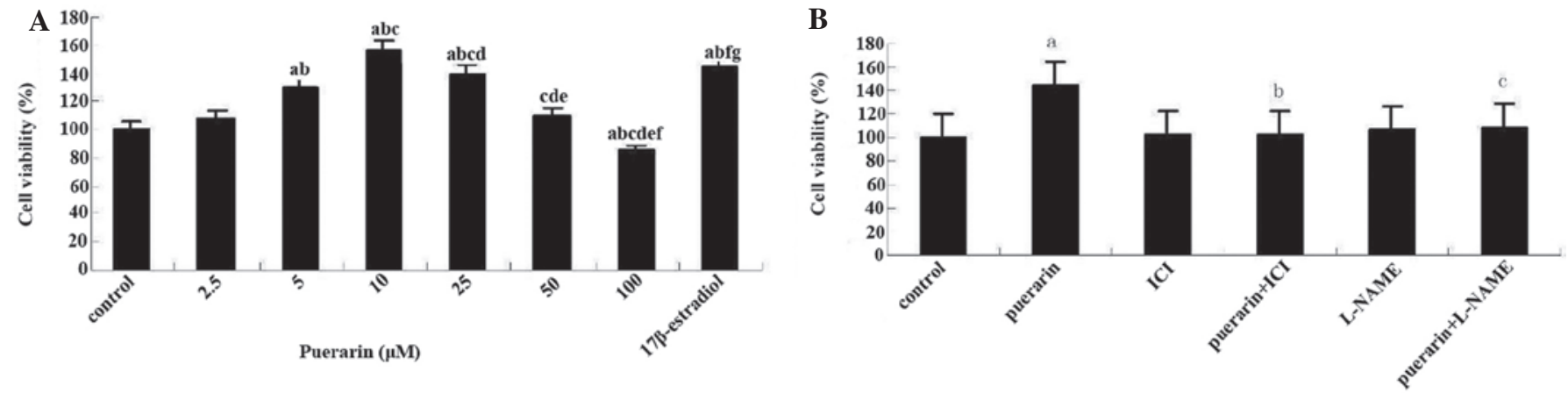

Figure 2. Effect of puerarin on cell proliferation in hBMSC cultures. (A) hBMSCs were treated with puerarin (2.5-100 $\mu \mathrm{M})$ for $48 \mathrm{~h}$, and cell viability was assessed using the MTT assay. Values are expressed as the percentage of the optical density of the control. ${ }^{a} \mathrm{P}<0.05$ vs. control group; ${ }^{b} \mathrm{P}<0.05 \mathrm{vs}$. puerarin $(2.5 \mu \mathrm{M})$ group; ${ }^{\mathrm{c}} \mathrm{P}<0.05$ vs. puerarin $(5.0 \mu \mathrm{M})$ group; ${ }^{\mathrm{d}} \mathrm{P}<0.05$ vs. puerarin $(10 \mu \mathrm{M})$ group; ${ }^{\mathrm{P}} \mathrm{P}<0.05$ vs. puerarin $(25 \mu \mathrm{M})$ group; ${ }^{\mathrm{f}} \mathrm{P}<0.05$ vs. puerarin $(50 \mu \mathrm{M})$ group; ${ }^{\mathrm{g}} \mathrm{P}<0.05$ vs. puerarin $(100 \mu \mathrm{M})$ group. (B) Cell viability of hBMSCs treated with puerarin $(2.5-100 \mu \mathrm{M})$ in the presence or absence of the estrogen receptor inhibitor ICI182780 $\left(10^{-7} \mathrm{M}\right)$ or the nitric oxide synthase inhibitor L-NAME $\left(6 \times 10^{-3} \mathrm{M}\right) .{ }^{a} \mathrm{P}<0.01 \mathrm{vs}$. control; ${ }^{b} \mathrm{P}<0.01 \mathrm{vs}$. puerarin $(10 \mu \mathrm{M})$ group. Values are expressed as the mean \pm standard deviation. hBMSC, human bone marrow stromal cell; ICI, ICI182780; L-NAME, Nx-nitro-L-arginine methyl ester.
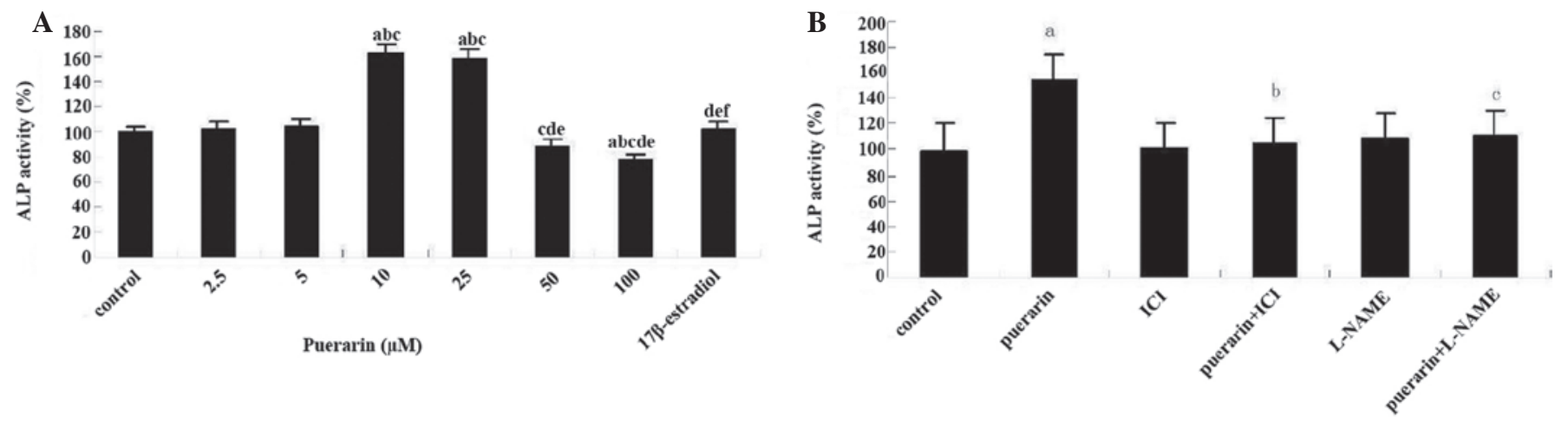

Figure 3. Effect of puerarin on ALP activity in hBMSC cultures. (A) After treatment with puerarin $(2.5-100 \mu \mathrm{M})$ or $17 \beta$-estradiol $\left(10^{-8} \mathrm{M}\right)$ for $48 \mathrm{~h}$, the supernatant of hBMSCs was used for the determination of ALP activity by measuring the release of $p$-nitrophenol with the ALP kit. Values are expressed as the percentage of the control. ${ }^{a} \mathrm{P}<0.05$ vs. control group; ${ }^{\mathrm{b}} \mathrm{P}<0.05$ vs. puerarin $(2.5 \mu \mathrm{M})$ group; ${ }^{\mathrm{C}} \mathrm{P}<0.05$ vs. puerarin $(5 \mu \mathrm{M})$ group; ${ }^{\mathrm{d}} \mathrm{P}<0.05 \mathrm{vs}$. puerarin $(10 \mu \mathrm{M})$ group; ${ }^{\mathrm{C}} \mathrm{P}<0.05$ vs. puerarin $(25 \mu \mathrm{M})$ group; ${ }^{\mathrm{f}} \mathrm{P}<0.05$ vs. puerarin $(100 \mu \mathrm{M})$ group. (B) ALP activity in hBMSCs treated with puerarin $(10 \mu \mathrm{M})$ in the presence or absence of ICI182780 $\left(10^{-7} \mathrm{M}\right)$ or L-NAME $\left(6 \times 10^{-3} \mathrm{M}\right)$ for eight days. The cellular ALP activity was assessed colorimetrically using $p$-nitrophenylphosphate as the substrate. Values are expressed as the mean \pm standard deviation of two experiments in triplicate. ${ }^{\mathrm{a}} \mathrm{P}<0.001$ vs. control group; ${ }^{\mathrm{b}} \mathrm{P}<0.01$, ${ }^{\mathrm{C}} \mathrm{P}<0.001 \mathrm{vs}$. puerarin $(10 \mu \mathrm{M})$. hBMSC, human bone marrow stromal cell; ICI, ICI182780; L-NAME, Nx-nitro-L-arginine methyl ester; ALP, alkaline phosphatase.

$24.5 \pm 1.2 \%(\mathrm{P}<0.05)$ following incubation with 10 and $25 \mu \mathrm{M}$ puerarin, while it was suppressed by $16.1 \pm 0.8 \%(\mathrm{P}<0.05)$ following incubation with $100 \mu \mathrm{M}$ puerarin. However, ALP activity was unaffected by $17 \beta$-estradiol. It is clearly shown that a dose of $10 \mu \mathrm{M}$ puerarin had the maximum effect on ALP activity (Fig. 3A). A previous study by our group identified a time-dependent increase in ALP activity and calcium deposition of hBMSCs in culture, which reached the highest levels after 8 and 12 days, respectively. Therefore, the present study determined these two parameters after 8 or 12 days of incubation with puerarin. As shown in Fig. 3A, puerarin $(2.5-100 \mu \mathrm{M})$ caused a dose-dependent increase in ALP activity at day eight, and the highest effect was obtained at a dose of $10 \mu \mathrm{M}$. Again, this elevation was clearly eliminated in the presence of the estrogen antagonist ICI182780 or the NOS inhibitor L-NAME (Fig. 3B). Treatment with ICI182780 or L-NAME alone had no effect on ALP activity.

Puerarin $(10 \mu M)$ increases mineralized nodule formation and calcium deposition in hBMSCs. The formation of mineralized nodules was observed in the present study. Alizarin red $\mathrm{S}$ staining indicated that mineral nodules were formed after 12 days of culture. Collagen I fibers and needle-like dense deposits, indicative of crystalline apatite structures, were obvious in these nodules (Fig. 4). Only a concentration of $10 \mu \mathrm{M}$ puerarin increased the number of mineral nodules formed, causing a $41 \pm 2.1 \%$ increase $(\mathrm{P}<0.05)$ in the number of nodules (Fig. 5A). This effect was slightly weaker than that of $17 \beta$-estradiol. By contrast, puerarin at higher concentrations markedly decreased bone nodule formation, with decreases of $17 \pm 0.3 \%(\mathrm{P}<0.05)$ at $50 \mu \mathrm{M}$ and $21 \pm 0.7 \%(\mathrm{P}<0.05)$ at $100 \mu \mathrm{M}$. In another experiment, assessment of calcium deposition showed that, as expected, $10 \mu \mathrm{M}$ puerarin caused the largest amount of calcium deposition (Fig. 5B). Furthermore, co-administration of puerarin $(10 \mu \mathrm{M})$ with ICI182780 or L-NAME abolished the increase in calcium deposition in the cultures following 12 days, while treatment with ICI182780 or L-NAME alone had no effect on calcium deposition (Fig. 5C).

Puerarin $(10 \mu M)$ enhances mRNA expression of Runx2, osterix and osteocalcin in hBMSCs. The effect of puerarin on the expression of Runx $2 /$ core-binding factor alpha 1 


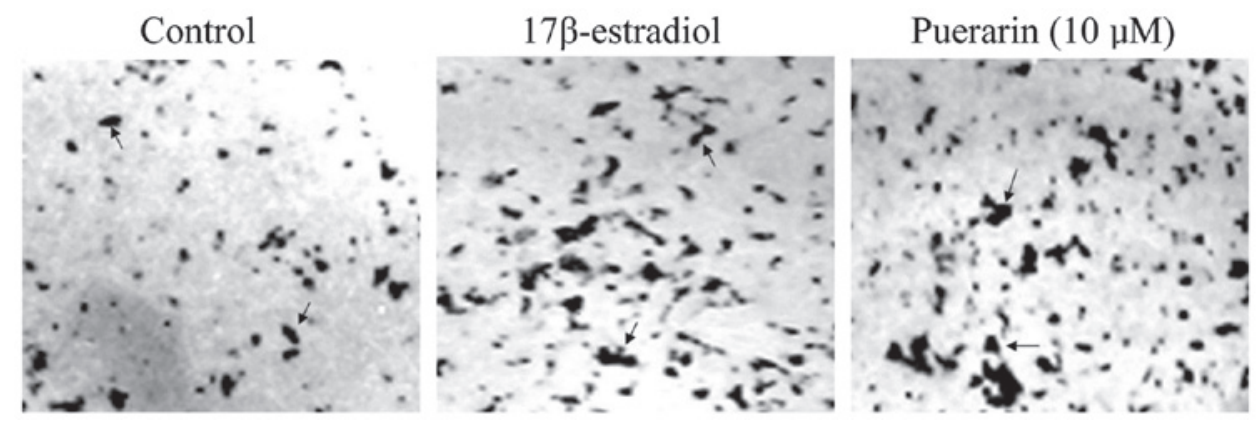

Figure 4. Effect of puerarin on mineral nodule formation in hBMSCs. hBMSCs were treated with puerarin $(10 \mu \mathrm{M})$ or $17 \beta$-estradiol $\left(10^{-8} \mathrm{M}\right)$ for 14 days, with the medium replaced every two days (containing $10 \%$ fetal bovine serum and the respective drug). The mineral nodules were stained with $0.1 \%$ alizarin red $\mathrm{S}$ and observed using a light microscope (magnification, $\mathrm{x} 40$ ). hBMSC, human bone marrow stromal cell.

A

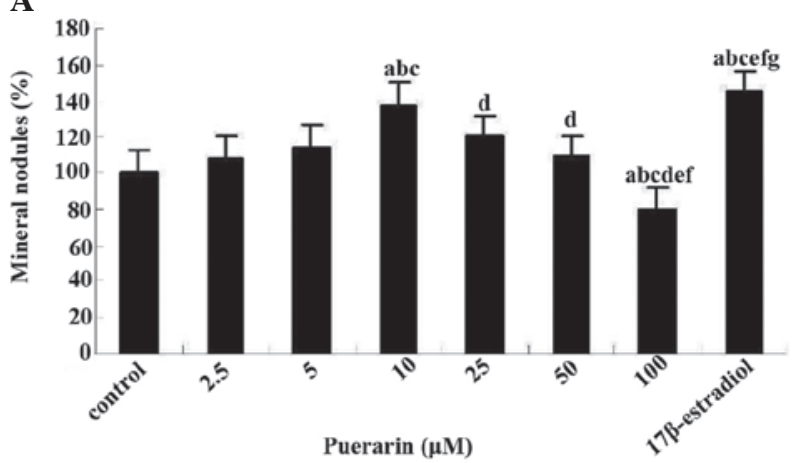

C

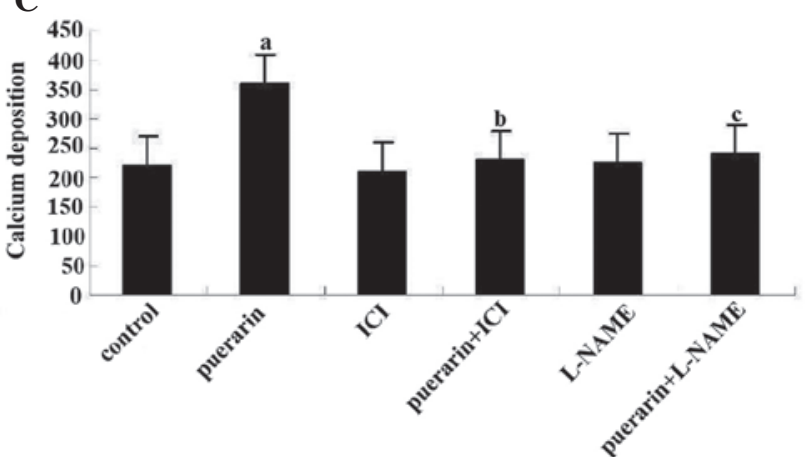

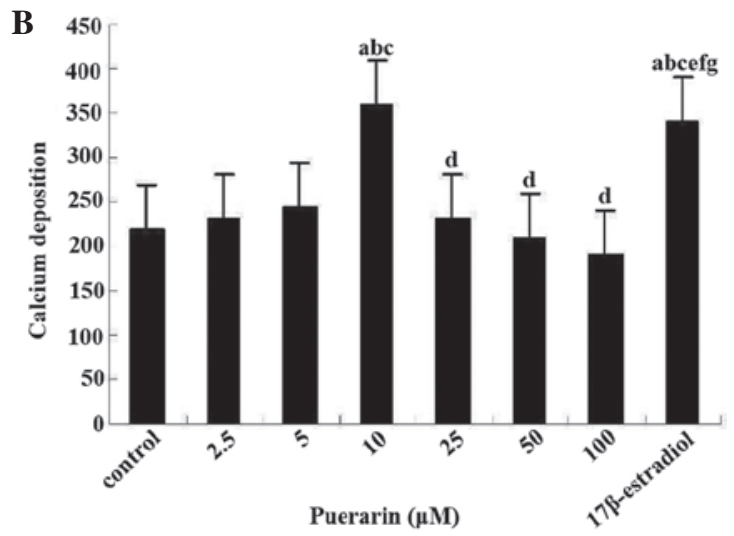

Puerarin $(\mu \mathrm{M})$

Figure 5. (A) Number of mineral nodules with a short diameter $>100 \mathrm{~mm}$ in 10 fields was counted. (B) Calcium deposition in hBMSC cultures treated with different concentrations of puerarin. Values are expressed as the percentage of the control. ${ }^{a} \mathrm{P}<0.05$ vs. control group; ${ }^{b} \mathrm{P}<0.05$ vs. puerarin $(2.5 \mu \mathrm{M})$ group; ${ }^{\mathrm{c}} \mathrm{P}<0.05$ vs. puerarin $(5.0 \mu \mathrm{M})$ group; ${ }^{\mathrm{d}} \mathrm{P}<0.05$ vs. puerarin $(10 \mu \mathrm{M})$ group; ${ }^{\mathrm{e}} \mathrm{P}<0.05$ vs. puerarin $(25 \mu \mathrm{M})$ group; ${ }^{\text {f }} \mathrm{P}<0.05$ vs. puerarin $(50 \mu \mathrm{M})$ group; ${ }^{g} \mathrm{P}<0.05$ vs. puerarin $(100 \mu \mathrm{M})$ group. (C) Calcium deposition in hBMSCs treated with puerarin $(10 \mu \mathrm{M})$ in the presence or absence of ICI182780 (10-7 M) or L-NAME $\left(6 \times 10^{-3} \mathrm{M}\right)$ for 12 days. Values are expressed as the mean \pm standard deviation. ${ }^{\mathrm{a}} \mathrm{P}<0.05$ vs. control. ${ }^{\mathrm{b}} \mathrm{P}<0.01,{ }^{\mathrm{C}} \mathrm{P}<0.001$ vs. puerarin (10 $\left.\mu \mathrm{M}\right)$ group. $\mathrm{hBMSC}$, human bone marrow stromal cell; ICI, ICI182780; L-NAME, Nx-nitro-L-arginine methyl ester.

(CBFA1), osterix and osteocalcin were evaluated by RT-qPCR analysis in RNA preparations from hBMSC cultures at day eight (Table I). Puerarin $(10 \mu \mathrm{M})$ significantly increased mRNA levels of Runx2/CBFA1 as well as its downstream genes osterix and osteocalcin as compared with those in the control. ICI182780 $\left(10^{-7} \mathrm{M}\right)$ or L-NAME $\left(6 \times 10^{-3} \mathrm{M}\right)$ reversed the puerarin-mediated upregulation of Runx $2 / \mathrm{Cbfa}$, osterix and osteocalcin. In addition, ICI182780 $\left(10^{-7} \mathrm{M}\right)$ or L-NAME $\left(6 \times 10^{-3} \mathrm{M}\right)$ alone had no effect on the expression of these genes, whereas E2 $\left(10^{-8} \mathrm{M}\right)$ served as a positive control and produced similar results to those of puerarin. These results were consistent with the previously observed NO/GMP signaling involved in puerarin- or E2-induced osteoblastic differentiation $(22,23)$.
Puerarin $(10 \mu M)$ increases the production of NO and cGMP in hBMSC cultures. Puerarin at concentrations of 2.5-10 $\mu \mathrm{M}$ dose-dependently enhanced NO levels in the culture media of BMSCs following 12 days, while these increases in NO production compared with the control faded with increasing doses of puerarin $(>10 \mu \mathrm{M})$ (Fig. 6A). This effect was abolished in the presence of ICI182780 or L-NAME (Fig. 6B). Assessment of cGMP formation in hBMSC cultures showed that only 10 and $25 \mu \mathrm{M}$ puerarin caused significant increases in cGMP levels, which were up to three-fold those in the $5 \mu \mathrm{M}$ puerarin group or the control group (Fig. 7A). This stimulating effect was completely inhibited by co-incubation with ICI182780 or L-NAME. Again, treatment with ICI182780 or 
Table I. Effect of estrogen receptor antagonist ICI182780 and nitrogen oxide synthase inhibitor L-NAME on the puerarin-induced mRNA expression of Runx2, osterix and osteocalcin of human bone marrow stromal cells by quantitative polymerase chain reaction.

\begin{tabular}{llll}
\hline Group & Runx2 & Osterix & Osteocalcin \\
\hline Control & $1.0 \pm 0.18$ & $1.0 \pm 0.16$ & $1.0 \pm 0.21$ \\
Puerarin $(10 \mu \mathrm{M})$ & $3.7 \pm 0.24^{\mathrm{a}}$ & $4.8 \pm 0.19^{\mathrm{a}}$ & $4.3 \pm 0.21^{\mathrm{a}}$ \\
ICI182780 & $1.0 \pm 0.24$ & $1.1 \pm 0.18$ & $0.9 \pm 0.24$ \\
Puerarin + ICI182780 & $1.1 \pm 0.22^{\mathrm{b}}$ & $1.2 \pm 0.19^{\mathrm{b}}$ & $1.0 \pm 0.16^{\mathrm{b}}$ \\
L-NAME & $0.9 \pm 0.13$ & $0.8 \pm 0.25$ & $1.1 \pm 0.24$ \\
Puerarin + L-NAME & $1.2 \pm 0.17^{\mathrm{b}}$ & $1.3 \pm 0.24^{\mathrm{b}}$ & $1.0 \pm 0.21^{\mathrm{b}}$ \\
$\mathrm{E}_{2}$ & $4.1 \pm 0.22^{\mathrm{a}}$ & $3.9 \pm 0.24^{\mathrm{a}}$ & $3.1 \pm 0.24^{\mathrm{a}}$ \\
$\mathrm{E}_{2}+\mathrm{ICI} 182780$ & $1.2 \pm 0.21^{\mathrm{c}}$ & $0.9 \pm 0.14^{\mathrm{c}}$ & $1.3 \pm 0.24^{\mathrm{c}}$ \\
\hline
\end{tabular}

Values are expressed as the mean \pm standard deviation. ${ }^{a} \mathrm{P}<0.01 \mathrm{vs}$. control; ${ }^{\mathrm{b}} \mathrm{P}<0.01 \mathrm{vs}$. puerarin; ${ }^{\mathrm{c}} \mathrm{P}<0.01 \mathrm{vs}$. $\mathrm{E}_{2}$. Runx 2 , Runt-related transcription factor 2; E, estrogen; L-NAME, Nx-nitro-L-arginine methyl ester.

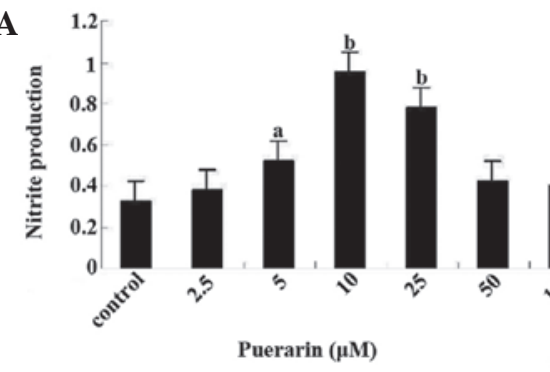

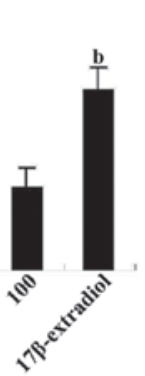

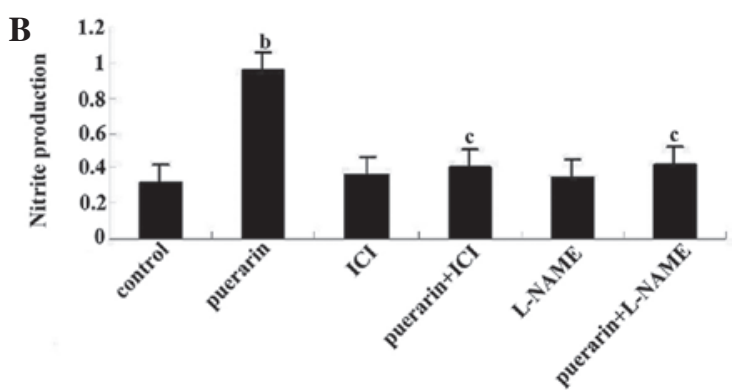

Figure 6. Effect of puerarin on nitrite content in the conditioned media of hBMSC cultures. (A) Dose-dependent effect of puerarin $(2.5-100 \mu \mathrm{M})$ on nitrite production by hBMSCs following 12 days. (B) Nitrite production by hBMSCs treated with puerarin $(10 \mu \mathrm{M})$ in the presence or absence of ICI182780 $\left(10^{-7} \mathrm{M}\right)$ or L-NAME $\left(6 \times 10^{-3} \mathrm{M}\right)$ following 12 days. Values are expressed as the mean \pm standard deviation of two experiments in triplicate. ${ }^{\mathrm{a}} \mathrm{P}<0.05$, ${ }^{\mathrm{b}} \mathrm{P}<0.01 \mathrm{vs}$. control; ${ }^{\mathrm{c}} \mathrm{P}<0.01$ vs. puerarin $(10 \mu \mathrm{M})$ group. hBMSC, human bone marrow stromal cell; ICI, ICI182780; L-NAME, Nx-nitro-L-arginine methyl ester.
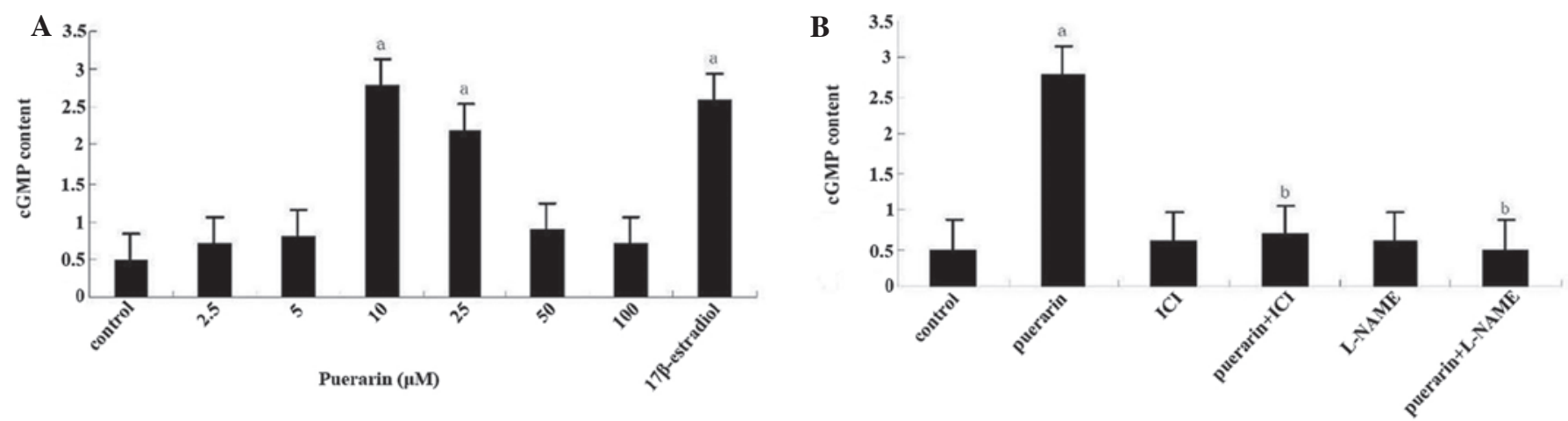

Figure 7. Effect of puerarin on cGMP content in hBMSC cultures. (A) Dose-dependent effect of puerarin (2.5-100 $\mu \mathrm{M})$ on cGMP content in hBMSC cultures following eight days. (B) cGMP content in hBMSC cultures with puerarin $(10 \mu \mathrm{M})$ in the presence or absence of ICI182780 $\left(10^{-7} \mathrm{M}\right)$ or L-NAME $\left(6 \times 10^{-3} \mathrm{M}\right)$ for eight days. The amount of cGMP was measured using an enzyme immunoassay kit. Values are expressed as the mean \pm standard deviation of two experiments performed in triplicate. ${ }^{a} \mathrm{P}<0.01 \mathrm{vs}$. control; ${ }^{\mathrm{b}} \mathrm{P}<0.001$ vs. puerarin $(10 \mu \mathrm{M})$ group. hBMSC, human bone marrow stromal cell; ICI, ICI182780; L-NAME, Nx-nitro-L-arginine methyl ester; cGMP, cyclic guanosine monophosphate.

L-NAME alone did not affect the cGMP content in the culture media (Fig. 7B).

\section{Discussion}

Estrogen has long been thought to only affect osteoclasts (24); however, it was recently shown that estrogen can also stimulate osteoblasts proliferation (25). Turners (26) hypothesized that estrogen can promote bone formation mainly by increasing the number of osteoblasts, which coincides with its effects on osteoporosis integrally. It is even thought that estrogen may act on the increased inducible NOS (iNOS) gene to gradually release NO and then regulate osteoblast activity $(27,28)$. Puerarin is a naturally occurring polyphenol that structur- 
ally resembles E2 and possesses estrogenic activity (29), suggesting that it may exert similar functions to those of E2 on NO synthesis and osteoblastic metabolism $(30,31)$.

The present study found that puerarin promotes osteoblastic anabolism in hBMSCs through the NO/cGMP/type II cGMP dependent protein kinase (PKG II) signaling pathway. It was demonstrated that puerarin dose-dependently elevated NO production as well as cGMP content in hBMSC cultures, which was associated with the stimulatory effects of puerarin on proliferation and osteoblastic differentiation of hBMSCs. Moreover, the puerarin-induced increase in NO levels was abolished by L-NAME, a non-selective NOS inhibitor, suggesting that puerarin has NO-releasing effects. This is consistent with the demonstrated protective effects of puerarin in the cardiovascular system (32).

NO is important for bone remodeling, as evidenced by in vitro and in vivo studies. NO synthesis in osteoblasts incubated with puerarin was mediated, in part, through the activation of endothelial NO synthase (eNOS) and increased iNOS expression $(33,34)$. NO produced in osteoblasts incubated with puerarin was shown to activate soluble guanylate cyclase, increasing intracellular cGMP, which activates soluble type I and membrane-bound type II PKG; other cGMP targets include phosphodiesterases and cyclic nucleotide-gated ion channels (35). PKG II-deficient mice are dwarfs due to a block in chondrocyte differentiation in bone growth plates, while PKG I-deficient mice have no obvious skeletal abnormalities (36). Pre-clinical and clinical studies supported osteogenic functions of $\mathrm{NO}$, although optimal dosing of $\mathrm{NO}$ and the potential for NO-induced oxidative stress may be problematic (37).

The present study identified that puerarin dose-dependently elevated NO production as well as cGMP/PKG II content in hBMSC cultures. However, puerarin at higher concentrations $(100 \mu \mathrm{M})$ remarkably decreased the NO/cGMP/PKG II content in cultured hBMSCs. The mechanisms underlying these processes require further study. Of note, L-NAME did not have any significant effect on control levels of nitrite production, although previous studies have shown an association between NO production, osteoblast differentiation and bone production.

In the present study, NO production and the anabolic effect of puerarin on hBMSCs were simultaneously blocked by ICI182780, an estrogen receptor (ER) antagonist, suggesting that puerarin exerts its function through ERs and that the NOS-NO pathway may include downstream effectors of the ER. These findings are consistent with other studies showing the stimulatory effects of puerarin on the osteoblastic cell line MC3T3-E1, which were also blocked by tamoxifen, another anti-estrogen reagent (38).

The present study demonstrated that puerarin, the major active component of the Traditional Chinese Medicine Radix Puerariae, potently induced osteoblastic differentiation markers, including ALP, Runx2, osterix and osteocalcin, and mineralization in hBMSCs. Upregulation of ALP, an enzyme serving as a marker of osteoblastic differentiation, occurs at the middle stage of differentiation. Puerarin, at concentrations $<10 \mu \mathrm{M}$, significantly increased ALP activity in a dose-dependent manner. Runx2, which belongs to the Runx family, is a key transcriptional modulator of osteoblast differentiation (39). Runx 2 has been shown to induce ALP activity, expression of bone matrix protein genes and mineralization in immature mesenchymal cells and osteoblastic cells in vitro (40). Osterix and osteocalcin (also known as the bone gla protein), which induce mesenchymal cells to differentiate into osteoblasts, are traditionally considered markers of osteoblast activity, as they are produced in osteoblasts and are associated with high bone turnover and increased bone mineral density (BMD) in a variety of clinical settings $(41,42)$. In the present study, puerarin $(10 \mu \mathrm{M})$ significantly increased mRNA expression of Runx2/CBFA1 as well as that of its downstream genes osterix and osteocalcin. The increase was slightly weaker than that caused by $17 \beta$-estradiol. Only a puerarin concentration of $10 \mu \mathrm{M}$ significantly affected the number of mineral nodules formed, causing a $\sim 38 \%$ increase in the number of nodules. By contrast, at the highest concentration of $100 \mu \mathrm{M}$, puerarin markedly decreased bone nodule formation. However, the mechanisms underlying these processes remain elusive.

In conclusion, the results of the present study indicated that puerarin, at concentrations $<10 \mu \mathrm{M}$, stimulated proliferation and osteoblastic differentiation of hBMSCs in a dose-dependent manner. This effect was mediated by estrogen receptors acting through the NO/cGMP/PKG II signaling pathway. However, puerarin at higher concentrations impaired osteoblastic proliferation and differentiation. The present study therefore suggested that puerarin may promote osteoblastic formation and effectively prevent post-menopausal osteoporosis. However, there is little research in vivo in the present, thus further experiments in an animal model will provide a foundation for clinical treatment of osteoporosis.

\section{Acknowledgements}

The present study was supported by a grant from the natural science foundation of Gansu Province in China (grant no. 1308RJZA232).

\section{References}

1. Corina M, Vulpoi C and Brănişteanu D: Relationship between bone mineral density, weight, and estrogen levels in pre and postmenopausal women. Rev Med Chir Soc Med Nat lasi 116: 946-950, 2012.

2. Han KO, Moon IG, Kang YS, et al: Nonassociation of estrogen receptor genotypes with bone mineral density and estrogen responsiveness to hormone replacement therapy in Korean postmenopausal women. J Clin Endocrinol Metab 82: 991-995, 1997.

3. Turner C: Hormone replacement therapy: its use in the management of acute menopausal symptoms. J Am Acad Nurse Pract 6: 318-320, 1994.

4. Palacios S, Christiansen C, Sánchez Borrego R, et al: Recommendations on the management of fragility fracture risk in women younger than 70 years. Gynecol Endocrinol 28: 770-786, 2012.

5. Lees B and Stevenson JC: The prevention of osteoporosis using sequential low-dose hormone replacement therapy with estradiol-17 beta and dydrogesterone. Osteoporos Int 12: 251-258, 2001.

6. Kaya C, Dinçer Cengiz S, Cengiz B and Akgün G: The long-term effects of low-dose 17 beta-estradiol and dydrogesterone hormone replacement therapy on $24 \mathrm{~h}$ ambulatory blood pressure in hypertensive postmenopausal women: a 1-year randomized, prospective study. Climacteric 9: 437-445, 2006.

7. Popp AW, Bodmer C, Senn C, et al: Prevention of postmenopausal bone loss with long-cycle hormone replacement therapy. Maturitas 53: 191-200, 2006.

8. Pike MC and Ross RK: Progestins and menopause: epidemiological studies of risks of endometrial and breast cancer. Steroids 65: 659-664, 2000. 
9. Boué SM, Wiese TE, Nehls S, et al: Evaluation of the estrogenic effects of legume extracts containing phytoestrogens. J Agric Food Chem 51: 2193-2199, 2003.

10. Chen $\mathrm{L}$ and Lu ZP: Randomized controlled trial of integrated traditional Chinese and western medicine treatment for posthepatitic cirrhotic ascites: a systematic review. Chin J Hepatol 19: 205-209, 2011 (In Chinese).

11. Lagari VS and Levis S: Phytoestrogens in the prevention of postmenopausal bone loss. J Clin Densitom 16: 445-449, 2013.

12. Chen Z, Lin SY, Zhou YH, et al: Analysis of clinical features of traditional Chinese medicine symptoms and syndromes of 220 patients with chronic aplastic anemia. Chin J Integr Tradit West Med 34: 43-45, 2014 (In Chinese).

13. Boué SM, Burow ME, Wiese TE, et al: Estrogenic and antiestrogenic activities of phytoalexins from red kidney bean (Phaseolus vulgaris L.). J Agric Food Chem 59: 112-120, 2011.

14. Wang Y, Wang WL, Xie WL, Li LZ, Sun J, Sun WJ and Gong HY: Puerarin stimulates proliferation and differentiation and protects against cell death in human osteoblastic MG-63 cells via ER-dependent MEK/ERK and PI3K/Akt activation. Phytomedicine 20: 787-796, 2013.

15. Armour KJ, Armour KE, van't Hof RJ, Reid DM, Wei XQ, Liew FY and Ralston SH: Activation of the inducible nitric oxide synthase pathway contributes to inflammation-induced osteoporosis by suppressing bone formation and causing osteoblast apoptosis. Arthritis Rheum 44: 2790-2796, 2001

16. Samuels A, Perry MJ, Gibson RL, Colley S and Tobias JH: Role of endothelial nitric oxide synthase in estrogen-induced osteogenesis. Bone 29: 24-29, 2001

17. Wang DH, Hu YS, Du JJ, et al: Ghrelin stimulates proliferation of human osteoblastic TE85 cells via NO/cGMP signaling pathway. Endocrine 35: 112-117, 2008.

18. El-Mowafy AM, Alkhalaf $M$ and Jaffal SM: Nongenomic activation of the GC-A enzyme by resveratrol and estradiol downstream from membrane estrogen receptors in human coronary arterial cells. Nutr Metab Cardiovasc Dis 17: 508-516, 2007.

19. Pan W, Quarles LD, Song LH, et al: Genistein stimulates the osteoblastic differentiation via NO/cGMP in bone marrow culture. J Cell Biochem 94: 307-316, 2005.

20. Andelman F, Kipervasser S, Maimon S, Fried I, Parmet Y and Neufeld MY: A revised intracarotid etomidate memory (Wada) procedure. Acta Neurol Scand 127: 97-102, 2013.

21. Hokazono E, Osawa S, Nakano T, et al: Development of a new measurement method for serum calcium with chlorophosphonazo-III. Ann Clin Biochem 46: 296-301, 2009.

22. Holzer G, Einhorn TA and Majeska RJ: Estrogen regulation of growth and alkaline phosphatase expression by cultured human bone marrow stromal cells. J Orthop Res 20: 281-288, 2002.

23. Almeida M, Martin-Millan M, Ambrogini E, et al: Estrogens attenuate oxidative stress and the differentiation and apoptosis of osteoblasts by DNA-binding-independent actions of the ERalpha. J Bone Miner Res 25: 769-781, 2010.

24. MacIntyre I, Zaidi M, Alam AS, Datta HK, Moonga BS, Lidbury PS, Hecker M and Vane JR: Osteoclast inhibition: an action of nitric oxide not mediated by cyclic GMP. Proc Natl Acad Sci USA 88: 2936-2940, 1991.

25. Galea GL, Meakin LB, Sugiyama T, et al: Estrogen receptor $\alpha$ mediates proliferation of osteoblastic cells stimulated by estrogen and mechanical strain, but their acute down-regulation of the Wnt antagonist Sost is mediated by estrogen receptor $\beta$. J Biol Chem 288: 9035-9048, 2013.
26. Turner RT, Evans GL and WakIey GK: Mechanism of action of estrogen on cancellous bone balance in tibiae of ovariectomized growing rat: Inhibition of indices of formation and resorption. J Bone Miner Res 8: 359-366, 1993.

27. Wang Q, Sun L, Zhang F, et al: Effects of $17 \beta$-estradiol on cell proliferation, cAMP and cGMP content and iNOS activity in human osteoblast-like osteosarcoma cell line TE85. Chin Pharmacol Bull 14: 314-317, 1998.

28. Cervellati C, Bonaccorsi G, Cremonini E, et al: Oxidative stress and bone resorption interplay as a possible trigger for postmenopausal osteoporosis. Biomed Res Int 2014: 563-569, 2014.

29. Manolagas SC, O'Brien CA and Almeida M: The role of estrogen and androgen receptors in bone health and disease. Nat Rey Endocrinol 9: 699-712, 2013

30. Michihara S, Tanaka T, Uzawa Y, Moriyama T and Kawamura Y: Puerarin exerted anti-osteoporotic action independent of estrogen receptor-mediated pathway. J Nutr Sci Vitaminol (Tokyo) 58: 202-209, 2012

31. Saha P, Saraswat G, Chakraborty P, Banerjee S, Pal BC and Kabir SN: Puerarin, a selective oestrogen receptor modulator, disrupts pregnancy in rats at pre-implantation stage. Reproduction 144: 633-645, 2012.

32. Deng Y, Ng ES, Yeung JH, et al: Mechanisms of the cerebral vasodilator actions of isoflavonoids of Gegen on rat isolated basilar artery. J Ethnopharmacol 139: 294-304, 2012.

33. Sheu SY, Tsai CC, Sun JS, Chen MH, Liu MH and Sun MG: Stimulatory effect of puerarin on bone formation through co-activation of nitric oxide and bone morphogenetic protein-2/mitogen-activated protein kinases pathways in mice. Chin Med J (Engl) 125: 3646-3653, 2012.

34. Brown JP, Dempster DW, Ding B, Dent-Acosta R, San Martin J, Grauer A, Wagman RB and Zanchetta J: Bone remodeling in postmenopausal women who discontinued denosumab treatment: off-treatment biopsy study. J Bone Miner Res 26: 2737-2744, 2011

35. Rangaswami HI, Marathe N, Zhuang S, et al: Type II cGMP-dependent protein kinase mediates osteoblast mechanotransduction. Handb Exp Pharmacol 191: 137-162, 2009.

36. Pfeifer A, Aszódi A, Seidler U, Ruth P, Hofmann F and Fässler R: Intestinal secretory defects and dwarfism in mice lacking cGMP-dependent protein kinase II. Science 274: 2082-2086, 1996.

37. Wimalawansa SJ: Rationale for using nitric oxide donor therapy for prevention of bone loss and treatment of osteoporosis in humans. Ann NY Acad Sci 1117: 283-297, 2007.

38. Yang F, Zhang R, He F, Wang XX, Zhao S and Yang G: Osteoblast response to puerarin-loaded porous titanium surfaces: an in vitro study. J Biomed Mater Res A 100: 1419-1426, 2012.

39. Wang N, Wang X, Cheng W, et al: Puerarin promotes osteogenesis and inhibits adipogenesis in vitro. Chin Med 8: 17, 2013

40. Brown JP, Delmas PD, Malaval L, Edouard C, Chapuy MC and Meunier PJ: Serum bone Gla-protein: a specific marker for bone formation in postmenopausal osteoporosis. Lancet 1: 1091-1093, 1984.

41. Minisola S, Rosso R, Romagnoli E, et al: Serum osteocalcin and bone mineral density at various skeletal sites: a study performed with three different assays. J Lab Clin Med 129: 422-429, 1997.

42. Koo KT, Lee SW, Lee MH, Kim KH, Jung SH and Kang YG: Time-dependent expression of osteoblast marker genes in human primary cells cultured on microgrooved titanium substrata. Clin Oral Implants Res 25: 714-722, 2014. 